\section{El Proyecto BRAIN: ¿Sólo se estudia en computadoras?}

\section{The BRAIN initiative: Is it only studied in computers?}

El progreso científico no justifica violar el bienestar de ningún individuo.

Claude Bernard, Padre de la medicina experimental.

\section{Sr. Editor:}

La iniciativa BRAIN, cuyas iniciales se pueden traducir como "Investigación del Cerebro a través del Avance de Neurotecnologías Innovadoras", es un billonario proyecto norteamericano de investigación para hacer un mapeo cerebral y conocer cómo interactúan las células cerebrales. Es una investigación que fue anunciada por el propio Presidente de los Estados Unidos de Norteamérica, Barack Obama ${ }^{1}$, y que, según los organizadores, dará a los científicos las herramientas necesarias para obtener una imagen del cerebro en acción y permitir comprender cómo pensamos, aprendemos o memorizamos, y ayudará a descubrir los secretos que esconden las enfermedades degenerativas del cerebro, como las enfermedades de Alzheimer, Parkinson y patologías psiquiátricas. En Europa, por otro lado, se ha desarrollado su análogo, el proyecto HUMAN BRAIN ${ }^{2}$, siendo uno de sus principales promotores el gobierno de Suiza, en cuya Escuela Politécnica Federal de Lausanne, labora el responsable del proyecto, el Profesor Dr. Henry Markram, un ingeniero de computadoras que, en julio de 2009, anunció públicamente que su equipo podrá crear el primer cerebro artificial en el año $2018^{3}$.

El proyecto HUMAN BRAIN es promocionado como un proyecto que va a ser estudiado en supercomputadoras. Pretende elaborar un modelo computarizado del cerebro humano que permita simular su funcionamiento en condiciones y situaciones extraordinarias. Sin embargo, la poca transparencia, el secretismo y autoritarismo de los organizadores del proyecto, la reducción drástica de neurocientíficos, hacen dudar a los expertos sobre sus verdaderos objetivos y condujo a que varios neurocientíficos se retiraran del proyecto en Europa y publicaran una carta de protesta en revistas científicas ${ }^{4}$.

Algunos científicos reciben con marcado escepticismo la idea de recrear un cerebro electrónicamente en sólo diez años. Para algunos tiene más que hacer con la ciencia ficción que con la ciencia, dado que actualmente ni siquiera pueden simular el sistema nervioso de animales simples ${ }^{4}$, peor aún la convicción de que van a curar enfermedades mediante el "mapeo" cerebral.

El Proyecto HUMAN BRAIN ha sido mal orientado y ha perdido apoyo de la comunidad neurocientífica, pues está sobreorientado a la ciencias de la computación. Aunque ha sido publicitado como neurociencia, la falla para incluir las opiniones de la mayoría de la comunidad neurocientífica es enorme y sorprendente; la pérdida de estudios de desarrollo, en humanos y animales, revela su debilidad científica ${ }^{4}$.

La ausencia de neurocientíficos es llamativa en un proyecto llamado precisamente "BRAIN", en que el líder del proyecto europeo es un ingeniero electrónico. Ciertamente, estudios científicos básicos de organismos modelos como ratas, monos moscas, pájaros o gusanos puedan contribuir más al entendimiento del cerebro humano que el tecnológico proyecto HUMAN BRAIN realizado en computadoras ${ }^{4}$. La comprensión de las interdependencias fisiológicas y patológicas en la biología del sistema nervioso debe ser la prioridad de la investigación; enfoques computacionales ciertamente pueden ser de ayuda pero no pueden reemplazar a los biológicos ${ }^{4}$.

Por otro lado, el proyecto norteamericano BRAIN $^{1}$ tiene entre sus principales promotores, la DARPA -Agencia de Proyectos de Investigación Avanzados de Defensa- una agencia del Departamento de Defensa de Estados Unidos de Norteamérica, lo que induce a pensar que su real objetivo es militar, y no precisamente mejorar la salud. Los nanodispositivos y el "mapeo" cerebral podrían ser utilizados para provocar tortura, robo de información y consecuente extorsión, y como arma para el control de la población civil. Existen antecedentes de que el ex Presidente norteamericano Bill Clinton admitió que el gobierno de Estados Unidos de Norteamérica realizaba experimentos humanos de control mental.

El enorme y casi utópico objetivo de "construir un cerebro humano", hace dudar de que la investigación sólo se realice en base a los estudios 


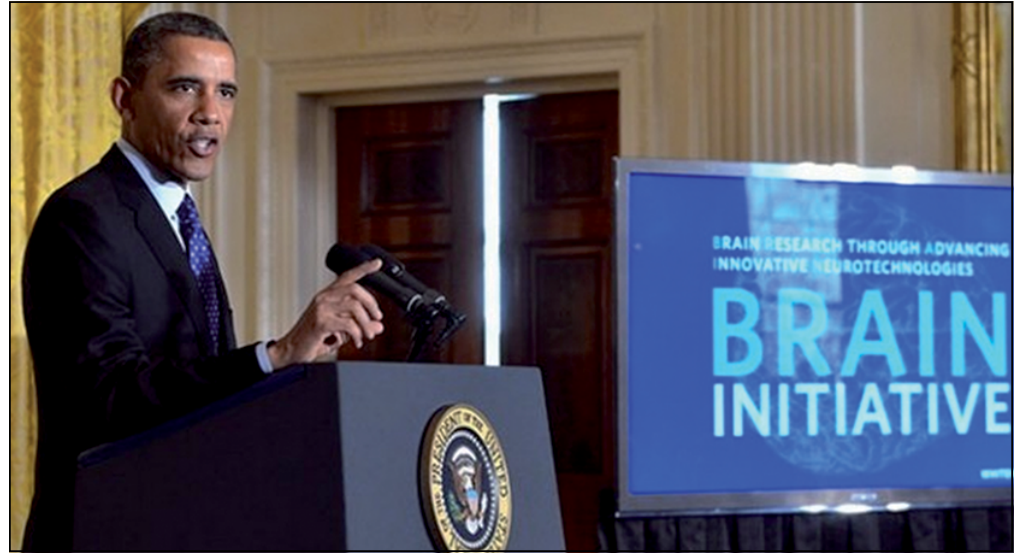

Figura 1. El Proyecto BRAIN. El Presidente de los Estados Unidos de Norteamérica Barack Obama anunciando el mapeo cerebral para mejorar la salud mundial. Fuente: http://www. whitehouse.gov/blog/2013/04/02/brain-initiative-challenges-researchers-unlock-mysteries-human-mind. en computadoras, dado que según los críticos, no existen hipótesis biológicas formuladas que sirvan de base para la simulación en computadoras ${ }^{5}$. No está claro en qué se ha invertido el dinero europeo en investigación cerebral en el proyecto HUMAN BRAIN $^{5}$.

El cerebro humano es el objeto más complejo en el universo. La enorme diferencia entre los objetivos casi utópicos de construir un cerebro artificial en sólo diez años y los medios, basados principalmente en computadoras, hacen sospechar que podría estarse desarrollando secretamente una investigación paralela con implantación secreta de nanodispositivos cerebrales en cerebros humanos, ilícita y forzadamente. Estos dispositivos estarían realizando el "mapeo" cerebral en seres humanos y sería la real fuente de información del proyecto BRAIN. Existen diversos escenarios y poblaciones donde podría estarse realizando esta ilícita experimentación, principalmente en países pobres.

En el mundo existen ejemplos deplorables de experimentación humana antiética de las potencias económicas, realizados en los países del tercer mundo, proyectos que son promocionados como lícitos, pero que el tiempo descubre que ocultaban una experimentación humana ilícita. La historia es, a grandes rasgos, repetitiva y los países, principalmente los del "tercer mundo" deben estar alertas ante posibles experimentaciones antiéticas.

David Salinas F. Sección Cardiología, Hospital Alberto Sabogal, ESSALUD

Cátedra de Medicina Humana, Universidad Nacional Mayor de San Marcos.

\section{Referencias}

1. The White House Brain Initiative. Brain research through advancing innovatives neurotechnologies. [Internet] [citado el 3 de marzo de 2015]. Disponible en: http://www.whitehouse.gov/share/brain-initiative.

2. European Comission Human Brain Proyect (Internet). [citado el 3 de marzo de 2015] Disponible en: https:// www.humanbrainproject.

3. Europa quiere construir un cerebro BBC Mundo 7 febrero de 2013[Internet] [citado el 3 de marzo de 2015]. Disponible en: http://www.bbc.co.uk/mundo/ noticias/2013/02/130201_tecnologia_cerebro_electronico_aa.

4. Open message to the European Commission concerning the Human Brain Project July 7, 2014 [Internet]. [citado el 3 de marzo de 2015] Disponible en: http:// neurofuture.eu.

5. Fregnax Y, Laurent G. Neuroscience: Where is the brain in the Human Brain Project? Nature 2014; 513 (7516): 27-9.

Correspondencia a:

David Salinas F.

Federico Villarreal 592, Urb. Ingeniería San Martín de Porres,

Lima, Perú.

dsalinas2009@yahoo.com

Conflictos de intereses: ninguno que declarar. 